\title{
Classificação de coeficientes de variação na experimentação com nutrição de eqüinos
}

\author{
[Classification of the coefficient of variation in experimentation with equine nutrition]
}

\author{
A.M.Q. Lana $^{1}$, J. Soares Neto ${ }^{2}$, F.Q. Almeida ${ }^{2}$, A.S.C. Rezende ${ }^{1}$, R.C. Prates ${ }^{1}$ \\ ${ }^{1}$ Escola de Veterinária - UFMG \\ Caixa Postal 567 \\ 31270-901 - Belo Horizonte, MG \\ ${ }^{2}$ Universidade Federal Rural do Rio de Janeiro - Seropédica, RJ
}

\begin{abstract}
RESUMO
Foi realizada a classificação de coeficientes de variação dos coeficientes de digestibilidade da matéria seca, matéria orgânica, proteína bruta, fibras em detergente neutro e ácido, energia bruta, fibra bruta, celulose, hemicelulose, lignina, extrato etéreo e ganho de peso diário, com base em artigos científicos e teses em eqüinocultura. Os coeficientes de variação foram classificados em: baixo $[\leq(\mathrm{m}-1 \mathrm{DP})]$; médio $[(\mathrm{m}-1 \mathrm{D})<\mathrm{CV} \leq(\mathrm{m}+1 \mathrm{DP})]$; alto $[(\mathrm{m}+1 \mathrm{DP})<\mathrm{CV} \leq(\mathrm{m}+2 \mathrm{DP})]$; e muito alto $[>(\mathrm{m}+2 \mathrm{DP})]$. Todas as respostas medidas apresentaram distribuição normal pelo teste Lilliefors. Quanto aos coeficientes de digestibilidade da fibra em detergente neutro e celulose, houve concordância entre a classificação proposta e a sugerida na literatura, sendo os mais discrepantes os coeficientes de digestibilidade da energia bruta e lignina. Desse modo, para cada variável analisada existe distribuição diferenciada dos coeficientes de variação, o que sugere a necessidade de classificação específica para as respostas medidas em nutrição de eqüinos.
\end{abstract}

Palavras-chave: eqüino, estatística, nutrição, precisão experimental

\begin{abstract}
Coefficients of variation $(\mathrm{CV})$ distribution for several variables in equine nutrition were obtained. The data are from theses and published scientific papers in equine nutrition. The $C V$ were classified as low $[C V \leq(m-1 s d)] ;$ medium $[(m-1 s d)<C V \leq(m+1 s d)] ;$ high $[(m+1 s d)<C V \leq(m+2 s d)]$ and very high $[C V>(m+2 s d)]$. The Lilliefors test for all variables showed no discrepancy from normal distribution. The distributions obtained for coefficients of digestibility for cellulose and fibre in neutral and acid detergent, were close enough to the classification suggested in the literature. However, the distributions for coefficient of digestibility for gross energy and lignin, showed high discrepancies from this classification suggesting that trial precision should be evaluated according to the variable considered. There is a different distribution for coefficients of variation for each variable suggesting the need of specific classification for each characteristic in equine studies.
\end{abstract}

Keywords: horse, statistic, nutrition, experimental precision

Recebido em 15 de julho de 2005

Aceito em 19 de abril de 2006

E-mail: lana@vet.ufmg.br

Apoio: FAPERJ-RJ 


\section{INTRODUÇÃO}

Para os pesquisadores que trabalham com a mesma resposta medida, o coeficiente de variação $(\mathrm{CV})$ pode ser um modo de se avaliar a precisão dos ensaios, pois, definido pela relação entre desvio-padrão (s) e média aritmética $(\bar{X})$, depende mais intensamente do desvio-padrão do que da média. Há um limite restrito na variação da média, enquanto o desvio-padrão pode sofrer pequena ou grande variação, dependendo da instabilidade provocada pela natureza da resposta medida (Sampaio, 2002).

Por ser medida relativa, o CV apresenta valores muito semelhantes em grande número de experimentos, se, em cada um desses, o desviopadrão for diretamente proporcional à produtividade média individual (Mead e Curnow, 1983). Esses autores enfatizaram que o pesquisador deve ter cuidado, pois o $\mathrm{CV}$ é medida sem sentido quando as observações experimentais envolvem valores positivos e negativos.

Segundo Gomes (1985), o CV dá idéia da precisão do experimento e, tendo-se por base os $\mathrm{CV}$ comumente encontrados em ensaios agrícolas de campo, eles são classificados em baixos, quando menores do que $10 \%$; médios, quando de 10 a $20 \%$; altos, quando de 20 a $30 \%$ e muito altos, quando maiores do que $30 \%$. Essa medida de precisão tem a vantagem de permitir a comparação entre experimentos, sem a necessidade de igualdade de unidades. Entretanto, Gomes (1991) salientou que o número de repetições influi nos valores do $\mathrm{CV}$, sobretudo pelo fato de que, mesmo havendo um experimento com coeficiente de variação maior em relação a outro, que, porém, possui menor número de repetições, aquele com maior coeficiente de variação seria mais preciso. O autor sugeriu como alternativa a utilização do índice de variação, que é a relação entre o $\mathrm{CV}$ e a raiz quadrada do número de repetições. Além disso, a classificação é muito abrangente e não leva em consideração particularidades da espécie estudada, e, principalmente, não faz distinção entre a natureza da instabilidade da resposta avaliada (Garcia, 1989; Scapim et al., 1995; Costa et al., 2002).

$\mathrm{Na}$ área agrícola, Scapim et al. (1995) verificaram, para cultura do milho, que os caracteres mais influenciados pelo ambiente apresentaram boa concordância entre a classificação dos CV sugerida por Gomes (1985) e Garcia (1989). Entretanto, para os caracteres menos influenciados pelo ambiente, o método proposto por Garcia (1989) seria o mais adequado.

Não há referência de valores de $\mathrm{CV}$ que identifique, especificamente em pesquisas com nutrição de eqüinos, o que poderia ser considerado, na avaliação da precisão de experimentos, a exemplo do que já foi sugerido nas culturas de eucalipto, forrageiras, milho, citros, suínos, arroz e soja (Garcia, 1989; Ambrosano e Schammas, 1994; Scapim et al., 1995; Amaral et al., 1997; Judice et al., 1999; Costa et al., 2002; Carvalho et al., 2003).

O coeficiente de variação estima a precisão experimental e é fundamental, uma vez que os resultados dos trabalhos científicos são, de modo geral, comparados. Assim, concluir, que os resultados de determinado trabalho são concordantes com os de outro autor pelo fato de ambos não detectarem efeito significativo, quanto à determinada fonte de variação, não será adequado se os coeficientes de variação forem muito discrepantes. Quando se compara o CV de determinada variável entre experimentos, devese considerar que os experimentos foram feitos em condições diferentes, que os ensaios podem diferir quanto à espécie e à variável em estudo; à transformação aplicada à variável; à heterogeneidade dos ambientes e dos animais utilizados, ao número de repetições e ao tamanho da parcela. Tais fatos reforçam a necessidade de referenciais de precisão dos experimentos conforme a natureza da resposta medida.

Este trabalho teve como objetivo avaliar os coeficientes de variação de experimentos na área de nutrição de eqüinos dada a inexistência de uma distribuição específica dos coeficientes de variação.

\section{MATERIAL E MÉTODOS}

Os dados foram obtidos por intermédio de revisão bibliográfica na Revista Brasileira de Zootecnia, no Arquivo Brasileiro de Medicina Veterinária e Zootecnia e na Pesquisa Agropecuária Brasileira, entre 1970 e 2002, e em 26 teses na área de nutrição de eqüinos. As 
variáveis analisadas foram ganho de peso diário (GPD), e os coeficientes de digestibilidade da matéria seca (CDMS), da matéria orgânica (CDMO), da proteína bruta (CDPB), da fibra em detergente neutro (CDFDN), da fibra em detergente ácido (CDFDA), da energia bruta (CDEB), da fibra bruta (CDFB), da celulose (CDCEL), da hemicelulose (CDHCEL), da lignina (CDLIG) e do extrato etéreo (CDEE), num total de 1569 observações (Tab. 1).

Tabela 1. Estatísticas descritivas: média geral ( $\bar{X})$, desvio-padrão (DP), máximo (Máx.), mínimo (Mín.), assimetria (As.), curtose (K.), testes Lilliefors (L.), assimetria (As=0) e curtose $(K=3)$ dos coeficientes de variação obtidos nos ensaios de nutrição de eqüinos

\begin{tabular}{|c|c|c|c|c|c|c|c|c|c|c|}
\hline Variável & $\mathrm{N}$ & $\bar{X}$ & DP & Máx. & Mín. & As. & $\mathrm{K}$. & L. & $\mathrm{As}=0$ & $\mathrm{~K}=3$ \\
\hline CDMS & 226 & 8,86 & 6,92 & 37,59 & 1,22 & 1,55 & 5,84 & $0,141^{\mathrm{ns}}$ & $0,32^{\mathrm{ns}}$ & $0,052^{\mathrm{ns}}$ \\
\hline CDMO & 159 & 9,12 & 5,52 & 18,90 & 2,15 & 0,29 & 1,48 & $0,199^{\mathrm{ns}}$ & $0,43^{\mathrm{ns}}$ & $0,055^{\mathrm{ns}}$ \\
\hline CDPB & 270 & 7,80 & 5,75 & 26,76 & 1,61 & 1,87 & 6,32 & $0,227^{\mathrm{ns}}$ & $0,24^{\mathrm{ns}}$ & $0,009 * *$ \\
\hline CDFDN & 116 & 13,63 & 8,32 & 36,22 & 1,23 & 0,80 & 3,19 & $0,162^{\mathrm{ns}}$ & $0,38^{\mathrm{ns}}$ & $0,44^{\mathrm{ns}}$ \\
\hline CDFDA & 139 & 16,52 & 9,52 & 39,55 & 2,04 & 1,03 & 3,25 & $0,188^{\mathrm{ns}}$ & $0,31^{\text {ns }}$ & $0,41^{\mathrm{ns}}$ \\
\hline CDEE & 42 & 11,33 & 9,22 & 24,01 & 0,80 & 0,33 & 1,16 & $0,236^{\mathrm{ns}}$ & $0,41^{\mathrm{ns}}$ & $0,016^{*}$ \\
\hline CDEB & 169 & 5,72 & 3,86 & 16,56 & 1,13 & 1,26 & 4,03 & $0,239^{\mathrm{ns}}$ & $0,26^{\mathrm{ns}}$ & $0,15^{\mathrm{ns}}$ \\
\hline CDFB & 162 & 15,89 & 11,4 & 31,37 & 6,30 & 0,47 & 1,06 & $0,314^{\mathrm{ns}}$ & $0,35^{\mathrm{ns}}$ & $0,009 * *$ \\
\hline CDHCEL & 93 & 11,28 & 10,2 & 37,42 & 2,47 & 1,48 & 4,14 & $0,238^{\mathrm{ns}}$ & $0,18^{\mathrm{ns}}$ & $0,088^{\mathrm{ns}}$ \\
\hline CDCEL & 102 & 15,85 & 7,37 & 30,40 & 4,61 & 0,04 & 1,95 & $0,108^{\mathrm{ns}}$ & $0,49^{\mathrm{ns}}$ & $0,15^{\mathrm{ns}}$ \\
\hline CDLIG & 38 & 41,81 & 15,6 & 68,90 & 25,7 & 0,67 & 1,74 & $0,306^{\mathrm{ns}}$ & $0,29^{\text {ns }}$ & $0,040 *$ \\
\hline GPD & 53 & 8,00 & 5,40 & 16,75 & 1,84 & 0,25 & 1,28 & $0,226^{\mathrm{ns}}$ & $0,44^{\mathrm{ns}}$ & $0,026 *$ \\
\hline Todas respostas & 1569 & 11,93 & 10,7 & 68,90 & 0,80 & 2,12 & 9,01 & $0,149^{* *}$ & $0,38^{\mathrm{ns}}$ & $0,040^{*}$ \\
\hline
\end{tabular}

CDMS, CDMO, CDPB, CDFDN, CDFDA, CDEE, CDEB, CDFB, CDHCEL, CDCEL e CDLIG = coeficientes de digestibilidade da matéria seca, matéria orgânica, proteína bruta, fibra em detergente neutro, fibra em detergente ácido, extrato etéreo, energia bruta, fibra bruta, hemicelulose, celulose e lignina, respectivamente. GPD = ganho de peso diário

Para análises estatísticas, utilizou-se o método de Kolmogorov-Smirnov, modificado por Lilliefors (1967) para avaliar a normalidade de distribuição dos dados e as estatísticas descritivas, média geral, desvio-padrão, máximo e mínimo; assimetria; probabilidade de assimetria $=0$; curtose; probabilidade de curtose $=3$ e tabelas de freqüências. Os coeficientes de variação foram classificados de acordo com a proposta de Garcia (1989), em: baixo $[\leq(\mathrm{m}-1 \mathrm{DP})]$; médio $[(\mathrm{m}-$ $1 \mathrm{DP})<\mathrm{CV} \leq(\mathrm{m}+1 \mathrm{DP})]$; alto $[(\mathrm{m}+1 \mathrm{DP})<\mathrm{CV} \leq(\mathrm{m}$ $+2 \mathrm{DP})]$; e muito alto $[>(\mathrm{m}+2 \mathrm{DP})]$.

Para a classificação dos coeficientes de variação, não foram especificados os delineamentos experimentais, considerando a conclusão de Estefanel et al. (1987), que sugerem que isso não influencia de modo significativo os valores dos coeficientes de variação, pois a forma de disposição do experimento visa, em princípio, atenuar a possibilidade de erro experimental.

\section{RESULTADOS E DISCUSSÕES}

Na Tab. 1 encontram-se os resultados dos testes Lilliefors, assimetria e curtose, e as estatísticas descritivas. Verifica-se, pelo teste Lilliefors, que os coeficientes de variação de todas as variáveis apresentaram distribuição não discrepante da normal. Observa-se, também, que as variáveis CDPB, CDEE, CDFB, CDLIG e GPD, apesar de apresentarem curtose diferente de 3, mostraram simetria. Para respostas estudadas, a menor amplitude foi obtida para o ganho de peso diário $(14,91)$ e a maior para o coeficiente de digestibilidade de lignina $(43,2)$. Com base nos valores de máximo e mínimo, nota-se grande amplitude dos dados entre e dentro das variáveis. Isso demonstra influência de grande número de fatores e justifica a necessidade de classificação específica dos coeficientes de variação referente a cada resposta medida.

Os intervalos dos coeficientes de variação segundo a classificação proposta por Garcia (1989) encontram-se na Tab. 2. No tocante às distribuições normais, $68,7 \%$ dos dados estariam incluídos entre m-1DP e m+1DP; 95,4\% dos dados incluídos entre m-2DP e m+2DP; e 99,7\% dos dados incluídos entre m-3DP e m+3DP. Para as distribuições normais ou aproximadamente normais, as percentagens acima podem ser mantidas ou aproximadamente mantidas, respectivamente (Spiegel, 1985). 
Classificação de coeficientes de variação...

Tabela 2. Distribuição dos valores de coeficientes de variação, nos ensaios com nutrição de eqüinos, segundo a classificação proposta

\begin{tabular}{ccccc}
\hline \multirow{2}{*}{$\begin{array}{c}\text { Resposta } \\
\text { Medida }\end{array}$} & \multicolumn{4}{c}{ Coeficiente de variação (\%) } \\
\cline { 2 - 5 } CDMS & $\mathrm{CV} \leq 1,94$ & Médio & $15,78<\mathrm{CV} \leq 22,7$ & Muito alto \\
CDMO & $\mathrm{CV} \leq 3,60$ & $1,94<\mathrm{CV} \leq 15,78$ & $\mathrm{CV}>22,7$ \\
CDPB & $\mathrm{CV} \leq 2,05$ & $3,60<\mathrm{CV} \leq 14,64$ & $14,64<\mathrm{CV} \leq 20,16$ & $\mathrm{CV}>20,16$ \\
CDFDN & $\mathrm{CV} \leq 5,31$ & $2,05<\mathrm{CV} \leq 13,55$ & $13,55<\mathrm{CV} \leq 19,30$ & $\mathrm{CV}>19,30$ \\
CDFDA & $\mathrm{CV} \leq 7,00$ & $5,31<\mathrm{CV} \leq 21,95$ & $21,95<\mathrm{CV} \leq 30,27$ & $\mathrm{CV}>30,27$ \\
CDEE & $\mathrm{CV} \leq 2,11$ & $7,00<\mathrm{CV} \leq 26,04$ & $26,04<\mathrm{CV} \leq 35,56$ & $\mathrm{CV}>35,56$ \\
CDEB & $\mathrm{CV} \leq 1,86$ & $2,11<\mathrm{CV} \leq 20,55$ & $20,55<\mathrm{CV} \leq 29,77$ & $\mathrm{CV}>29,77$ \\
CDFB & $\mathrm{CV} \leq 4,49$ & $1,86<\mathrm{CV} \leq 9,58$ & $\mathrm{CV}>13,44$ \\
CDHCEL & $\mathrm{CV} \leq 1,10$ & $4,49<\mathrm{CV} \leq 27,29$ & $27,29<\mathrm{CV} \leq 38,69$ & $\mathrm{CV}>38,69$ \\
CDCEL & $\mathrm{CV} \leq 8,48$ & $1,10<\mathrm{CV} \leq 21,46$ & $21,46<\mathrm{CV} \leq 31,64$ & $\mathrm{CV}>31,64$ \\
CDLIG & $\mathrm{CV} \leq 26,20$ & $8,48<\mathrm{CV} \leq 23,22$ & $23,22<\mathrm{C} \leq 30,59$ & $\mathrm{CV}>30,59$ \\
GPD & $\mathrm{CV} \leq 2,60$ & $26,20<\mathrm{CV} \leq 57,42$ & $57,42<\mathrm{CV} \leq 73,03$ & $\mathrm{CV}>73,03$ \\
\hline Classificação de & $\mathrm{CV} \leq 10,00$ & $2,60<\mathrm{CV} \leq 13,40$ & $13,40<\mathrm{CV} \leq 18,80$ & $\mathrm{CV}>18,80$ \\
Gomes (1985) & \multirow{2}{*}{$10,00<\mathrm{CV} \leq 20,00$} & $20,00<\mathrm{CV} \leq 30,00$ & $\mathrm{CV}>30,00$
\end{tabular}

CDMS, CDMO, CDPB, CDFDN, CDFDA, CDEE, CDEB, CDFB, CDHCEL, CDCEL e CDLIG = coeficientes de digestibilidade da matéria seca, matéria orgânica, proteína bruta, fibra em detergente neutro, fibra em detergente ácido, extrato etéreo, energia bruta, fibra bruta, hemicelulose, celulose e lignina, respectivamente. GPD = ganho de peso diário

A variável CDEB apresentou limites para os intervalos de coeficientes de variação menores que as demais variáveis $(\mathrm{CV} \leq 1,9 \%$; $1,9 \%<\mathrm{CV} \leq 9,6 \% ; 9,6 \%<\mathrm{CV} \leq 13,4 \%$ e $\mathrm{CV}>13,4 \%$ , para CV baixo, médio, alto e muito alto, respectivamente), enquanto a variável CDLIG apresentou os maiores valores $(\mathrm{CV} \leq 26,2 \%$; $26,2 \%<\mathrm{CV} \leq 57,4 \% ; \quad 57,4 \%<\mathrm{CV} \leq 73,0 \% \quad$ e $\mathrm{CV}>73,0 \%$, para $\mathrm{CV}$ baixo, médio, alto e muito alto, respectivamente). O coeficiente de digestibilidade de lignina apresentou consideráveis diferenças em razão da estrutura da parede celular das plantas dentro das espécies, decorrentes da idade, do clima e da disponibilidade de nutrientes, e diferenças entre as espécies forrageiras. Além disso, os métodos de determinação das ligninas são suscetíveis a erros na mensuração da resposta, como aquecimento nas várias etapas de determinação (Almeida, 1995). Os resultados obtidos por Almeida (1995) indicam coeficientes de digestibilidade de ligninas que variam de -8, 4, 6,3 e $40,9 \%$ para os volumosos coast-cross, napier e alfafa, respectivamente. A variável CDCEL e a CDFDA apresentaram distribuição de valores de coeficientes de variação mais próximas da classificação proposta por Gomes (1985). Portanto, verificou-se que as faixas de distribuição dos coeficientes de variação apresentaram valores específicos, o que, segundo Kalil (1977), mostra que, na avaliação dos valores de $\mathrm{CV}$, a utilização da sugestão de Gomes (1985), usualmente, feita pelos pesquisadores, leva a resultados divergentes dos encontrados para todas as respostas medidas.

Quando foram analisados todos os dados ao mesmo tempo, independente da resposta medida, não se verificou a normalidade dos dados (Tab. 1), reforçando a importância do estudo por variável, conforme recomendação de Federer (1957), Kalil (1977) e Steel e Torrie (1980).

As freqüências esperadas e observadas, segundo a classificação sugerida, bem como as freqüências observadas segundo a classificação proposta por Gomes (1985) estão apresentadas na Tab. 3. As freqüências esperadas foram obtidas considerando-se que os coeficientes de variação encontrados na experimentação com nutrição de eqüinos tenham distribuição normal ou aproximadamente normal. Sob normalidade, tem-se a expectativa de que a maioria dos experimentos apresente coeficientes de variação próximos da média e de que coeficientes de variação extremamente baixos ou extremamente altos ocorram, porém com baixa freqüência. 
Tabela 3. Freqüências dos coeficientes de variação, segundo as classificações propostas por Garcia (1989) e Gomes (1985), a partir dos dados obtidos nos ensaios de nutrição de eqüinos

\begin{tabular}{|c|c|c|c|c|c|c|c|c|}
\hline \multirow{3}{*}{ Variável } & \multicolumn{8}{|c|}{ Classificação dos coeficientes de variação (\%) } \\
\hline & \multicolumn{4}{|c|}{ Garcia (1989) } & \multicolumn{4}{|c|}{ Gomes (1985) } \\
\hline & Baixo & Médio & Alto & Muito alto & Baixo & Médio & Alto & Muito alto \\
\hline CDMS & 7,25 & 79,71 & 10,14 & 2,89 & 66,67 & 21,74 & 10,14 & 1,45 \\
\hline $\mathrm{CDMO}$ & 13,33 & 66,67 & 15,97 & 4,03 & 53,33 & 46,67 & 0,00 & 0,00 \\
\hline CDPB & 2,5 & 82,5 & 7,50 & 5,00 & 80,00 & 15,00 & 5,00 & 0,00 \\
\hline CDFDN & 23,00 & 43,67 & 29,01 & 4,32 & 33,33 & 47,22 & 13,89 & 5,56 \\
\hline CDFDA & 9,09 & 72,73 & 9,09 & 9,09 & 22,73 & 54,54 & 9,09 & 13,64 \\
\hline CDEE & 5,88 & 84,74 & 5,88 & 3,50 & 55,56 & 11,11 & 33,33 & 0,00 \\
\hline CDEB & 10,53 & 73,68 & 10,53 & 5,26 & 84,21 & 15,79 & 0,00 & 0,00 \\
\hline CDFB & 15,01 & 66,67 & 18,36 & 0,00 & 33,33 & 33,33 & 16,67 & 16,67 \\
\hline CDHCEL & 11,02 & 70,8 & 9,09 & 9,09 & 54,54 & 27,28 & 9,09 & 9,09 \\
\hline CDCEL & 18,77 & 68,42 & 10,53 & 2,28 & 26,32 & 36,84 & 31,58 & 5,26 \\
\hline CDLIG & 16,67 & 66,67 & 15,10 & 1,57 & 0,00 & 0,00 & 16,67 & 83,33 \\
\hline GPD & 9,09 & 63,64 & 21,67 & 5,60 & 54,55 & 45,45 & 0,00 & 0,00 \\
\hline
\end{tabular}

Freqüênci $15,86 \quad 68,27 \quad 13,59 \quad 2,28$

a esperada

CDMS, CDMO, CDPB, CDFDN, CDFDA, CDEE, CDEB, CDFB, CDHCEL, CDCEL e CDLIG = coeficientes de digestibilidade da matéria seca, matéria orgânica, proteína bruta, fibra em detergente neutro, fibra em detergente ácido, extrato etéreo, energia bruta, fibra bruta, hemicelulose, celulose e lignina, respectivamente. GPD = ganho de peso diário

Observa-se na Tab. 3 que não há discrepância relevante na distribuição de freqüência encontrada nas categorias de CV baixo, médio, alto e muito alto pelo método proposto por Garcia (1989) e as freqüências esperadas. Notam-se freqüências mais altas, na categoria de coeficiente de variação médio, para as variáveis CDPB e CDEE, iguais a 82,5 e $84,7 \%$, respectivamente. Esses resultados culminaram em redução das freqüências observadas das demais classes (CV baixo, alto e muito alto), para ambas as variáveis. Fato contrário a esse foi observado para a variável CDFDN, em que as freqüências observadas foram iguais a 23,00; 43,$67 ; 29,01$ e $4,23 \%$ nas categorias de CV baixo, médio, alto e muito alto, respectivamente.

$\mathrm{Na}$ distribuição de valores de coeficientes de variação (Tab. 3) baseada na classificação recomendada por Gomes (1985), verifica-se que os valores encontrados na literatura consultada, para a maioria das variáveis, enquadram-se mais freqüentemente nas categorias de $\mathrm{CV}$ baixo, principalmente, seguidas pela categoria de $\mathrm{CV}$ médio. Para as variáveis CDMO, CDEB e GPD, nenhum dos valores seria classificado como $\mathrm{CV}$ alto ou muito alto. Entretanto, para a variável CDLIG, 16,7 e $83,3 \%$ dos valores obtidos seriam classificados como altos e muito altos, respectivamente e nenhum dos dados seria classificado como baixo ou médio. Esses resultados concordam com Scapim et al. (1995) que verificaram, em estudos para a cultura do milho, freqüências maiores nas categorias de $\mathrm{CV}$ baixo ou médio quando submetidos à classificação sugerida por Gomes (1985).

As distribuições de freqüências obtidas apresentaram um afastamento maior em relação à freqüência esperada, quando comparado ao método proposto por Garcia (1989). Estes resultados sugerem que, para outras respostas estudadas em pesquisas com eqüinos, a classificação recomendada por Gomes (1985) pode não ser a mais adequada, sendo necessária análise mais específica para outros caracteres.

\section{CONCLUSÕES}

Para cada variável, dentro da nutrição eqüina, existe distribuição específica de valores de coeficientes de variação; o coeficiente de digestibilidade de energia bruta foi o que apresentou menor instabilidade relativa (CV); enquanto o coeficiente de digestibilidade da lignina a maior instabilidade relativa. Para os caracteres do coeficiente de digestibilidade da 
fibra em detergente neutro, extrato etéreo, hemicelulose e celuloses, não houve discrepância relevante entre a classificação de $\mathrm{CV}$ proposta no presente trabalho com a literatura pertinente.

\section{REFERÊNCIAS BIBLIOGRÁFICAS}

ALMEIDA, M.I.V.; FERREIRA, W.M.; ALMEIDA, F.Q. et al. Predição da energia digestível de dietas para eqüinos a partir de seu conteúdo fibroso. Arq. Bras. Med. Vet. Zootec., v. 47, p. 699-705, 1995.

AMARAL, A.M.; MUNIZ, J.A.; SOUZA, M. Avaliação do coeficiente de variação como medida da precisão na experimentação com citros. Pesq. Agropec. Bras., v.32, p.1221-1225,1997.

AMBROSANO, G.M.B.; SCHAMMAS, E.A. Avaliação dos coeficientes de variação em experimentos com forrageiras. Bol. Ind. Anim., v.51, p.13-20, 1994.

CARVALHO, C.G.P.; ARIAS, C.A.A.; TOLEDO, J.F.F. et al. Proposta de classificação dos coeficientes de variação em relação à produtividade e altura da planta de soja. Pesq. Agropec. Bras., v.38, p.187-193, 2003.

COSTA, N.H.A.D.; SERAPHIN, J.C.; ZIMMERMANN, F.J.P. Novo método de classificação de coeficientes de variação para a cultura do arroz de terras altas. Pesq. Agropec. Bras., v.37, p.243-249, 2002.

ESTEFANEL, V.; PIGNATARO, I.A.B.; STORCK, L. Avaliação do coeficiente de variação de experimentos com algumas culturas agrícolas. In: SIMPÓSIO DE ESTATÍSTICA APLICADA À EXPERIMENTAÇÃO AGRONÔMICA, 2., 1987, Londrina. Anais... Londrina: Universidade Estadual de Londrina, 1987. p.115-131.
FEDERER, W. T. Experimental design. New York: J. Wiley, 1957. 611p.

GARCIA, C.H. Tabelas para classificação do coeficiente de variação. Piracicaba: IPEF, 1989. 12p. (Circular técnica, 171).

GOMES, F.P. Curso de estatística experimental. 13.ed. São Paulo: ESALQ/USP, 1985. 467p.

GOMES, F.P. $O$ índice de variação, um substituto vantajoso do coeficiente de variação. Piracicaba: IPEF, 1991.4p. (Circular técnica, 178).

JUDICE, M.G.; MUNIZ, J.A.; CARVALHEIRO, R. Avaliação do coeficiente de variação na experimentação com suínos. Cienc. Agrotecnol., v.23, p.170-173, 1999.

KALIL, E. B. Principios de técnica experimental com animais. Piracicaba: ESALQ/USP, 1977. 210p.

LILLIEFORS, H.W. On the Kolmogorov-Smirnov test for normality with mean and variance unknown. J. Am. Stat. Assoc., v.62, p.399-402, 1967.

MEAD, R.; CURNOW, R.N. Statistical methods in agriculture and experimental biology. New York: Chapman and Hall, 1983. 335 p.

SAMPAIO, I.B.M. Estatística aplicada à experimentação animal. 2.ed. Belo Horizonte: FEPMVZ, 2002. 265p.

SCAPIM, C.A.; CARVALHO, C.G.P.; CRUZ, C.D. Uma proposta de classificação dos coeficientes de variação para a cultura do milho. Pesq. Agropec. Bras., v.30, p.683-686,1995.

SPIEGEL, M. R. Estatística. São Paulo: Mc GrawHill, 1985. 454p.

STEEL, R.G.D.; TORRIE, J.H. Principles and procedures of statistics: with reference to the biological sciences. New York: Mc Graw-Hill, 1980. 633p. 